\title{
Diagnosis and management challenge of a granular cell astrocytoma of the pineal region: case report
}

\author{
Abad Cherif El Asri, MD, ${ }^{1}$ Hassan Baallal, MD, ${ }^{1}$ Youssef Zoubeir, MD, ${ }^{2}$ Mohamed Sinaa, MD, ${ }^{2}$ \\ Abderrahman Albouzidi, MD, ${ }^{2}$ Miloudi Gazzaz, MD, ${ }^{1}$ Ali Akhaddar, MD, ${ }^{1}$ Mohamed Boucetta, MD, ${ }^{1}$ \\ and Brahim El Mostarchid, MD'1
}

Departments of ${ }^{1}$ Neurosurgery and ${ }^{2}$ Pathology, Military Hospital, Rabat, Morocco

\begin{abstract}
Granular cell astrocytoma (GCA) is a rare type of infiltrative brain tumor with most reported cases occurring in the suprasellar region. A pineal localization is extremely rare, with only 4 previously reported cases in the literature. The authors describe the case of a 16-year-old boy who developed signs of increased intracranial pressure and Parinaud syndrome. Cranial CT and MRI revealed a well-demarcated and enhanced mass in the pineal region accompanied by obstructive hydrocephalus. Subtotal resection was performed via a subtemporal approach. A histological diagnosis of GCA was made. Three years after surgery, the patient was alive and well without adjuvant therapy, and serial MRI showed no signs of progression of a small residual tumor. After a thorough review of the different epidemiological, clinical, and imaging features; treatments; and prognoses of GCAs in other intracranial localizations, the authors analyzed features of this tumor in the pineal region.
\end{abstract}

http://thejns.org/doi/abs/10.3171/2014.10.PEDS13388

KEY WORDS granular cell tumor; astrocytoma; pineal region; surgery; oncology

$\mathrm{P}$ RIMARY pineal region tumors are rare, with an estimated incidence of approximately $1 \%$ of all intracranial neoplasms. They are more common in children than adults. ${ }^{8,10}$ In general, the most common pineal region tumors are germ cell and pineal parenchymal tumors. On the other hand, a tumor can arise from glial components of the pineal gland, including ganglioneuromas, gangliogliomas, and gliomas ${ }^{6,8}$ Granular cell astrocytoma (GCA) is thought to represent an unusual astrocytoma originating from the pineal gland. ${ }^{3,10}$ There have been only 4 previously reported cases of GCA in the pineal region. ${ }^{9,10,15}$ In this report, we describe a surgical case of GCA in the pineal region. After a thorough review of the different epidemiological, clinical, and imaging features; treatments; and prognoses of GCAs in other intracranial localizations, we analyzed features of this GCA in the pineal region (Table 1).

\section{Case Report}

History and Examination

A 16-year-old previously healthy boy presented with a 1-month history of excruciating headaches associated with vertigo and occasional episodes of nausea and vomiting. He had abnormal extraocular movements with incomplete Parinaud syndrome and moderate bilateral papilledema. There was no other neurological deficit. Cranial CT demonstrated a mass of the pineal region with third ventricle obstruction resulting in hydrocephalus. Magnetic resonance imaging was performed, and the T1-weighted sequence revealed a low-intensity lesion of the pineal body and posterior third ventricle wall, measuring $3 \times 3 \times 4 \mathrm{~cm}$, with intense and homogeneous enhancement with Gd (Fig. 1). Serum levels of human chorionic gonadotropin, $\alpha$-fetoprotein, carcinoembryonic antigen, and placental alkaline phosphatase were negative.

\section{Treatment}

After ventriculoperitoneal shunt surgery was performed, the patient's symptoms disappeared except for the intermittent diplopia. One month later, the pineal tumor was surgically treated using the left subtemporal approach. At surgery, the tumor was reddish, soft, and vascular rich.

ABBREVIATIONS EMA = epithelial membrane antigen; GCA = granular cell astrocytoma; $\mathrm{GCT}$ = granular cell tumor; GFAP = glial fibrillary acidic protein . SUBMITTED July 26, 2013. ACCEPTED October 17, 2014.

INCLUDE WHEN CITING Published online February 20, 2015; DOI: 10.3171/2014.10.PEDS13388.

DISCLOSURE The authors report no conflict of interest concerning the materials or methods used in this study or the findings specified in this paper. 


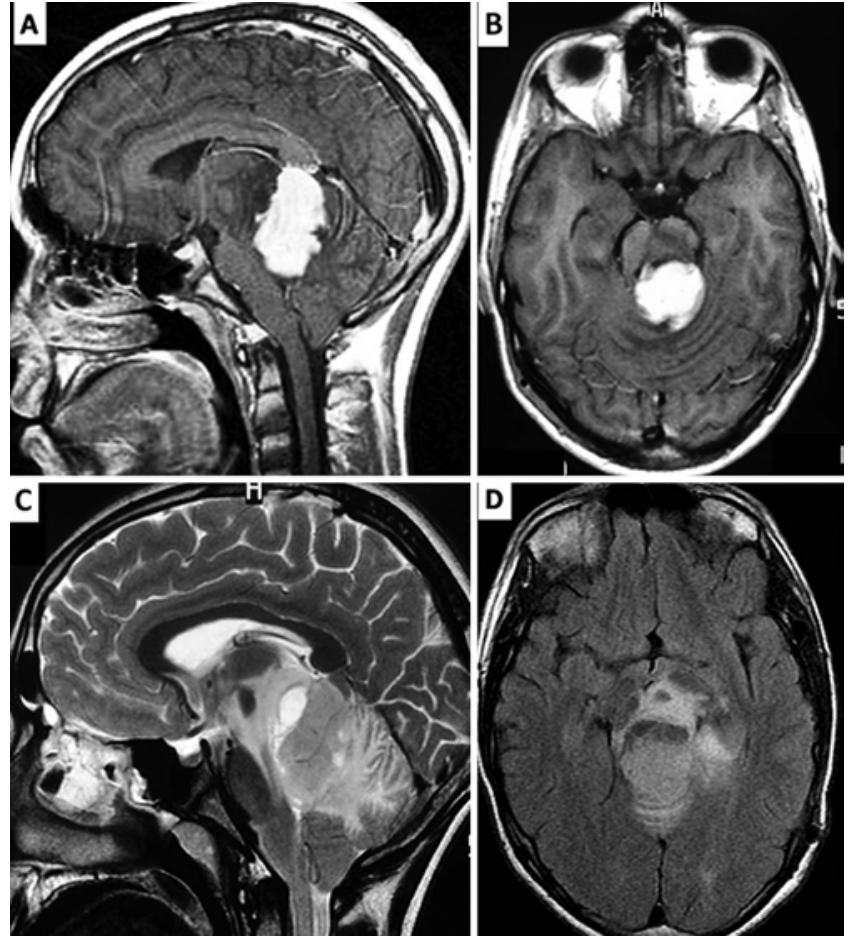

FIG. 1. Preoperative sagittal (A) and axial (B) T1-weighted MR images showing a $3 \times 3 \times 4-\mathrm{cm}$ homogeneously enhanced lesion in the pineal body and third ventricle wall. Sagittal T2-weighted MR image (C) and axial FLAIR image (D) showing hyperintensity of the adjacent structures.

Approximately $70 \%$ of the tumor was resected; the rest was closely apposed to the basal and internal veins.

\section{Posttreatment Course}

Histological examination showed the specimen to be moderately cellular with marked nuclear pleomorphism and foamy cytoplasm. Numerous bizarre multinucleated giant cells but no mitosis, necrosis, or endothelial proliferation were observed (Fig. 2). Glial fibrillary acid protein (GFAP), epithelial membrane antigen (EMA), macrophage marker CD68, PAS, and S100 were immunohistochemically positive. The tumor cells were negative for PAS-diastase, anti-neurofilaments, human chorionic gonadotropin, $\alpha$-fetoprotein, and placental alkaline phosphatase antibodies. Consequently, the tumor proved to be a GCA.

Postoperatively, mild right paresis was newly observed, but there was no other neurological deficit, and the patient did not require care in his daily life. Because the tumor was considered benign, he did not receive any adjuvant therapies. Cranial MRI studies at 6 months, 1 year, and 3 years after treatment showed no signs of growth of the residual tumor (Fig. 3).

\section{Discussion}

Granular cell astrocytoma, which has also been called "intracerebral granular cell tumor" or "astrocytoma with granular cell differentiation," is a rare type of infiltrative brain tumor. It was first described by von Luthy and Klinger, in 1951, as a granular cell tumor (GCT) in the 


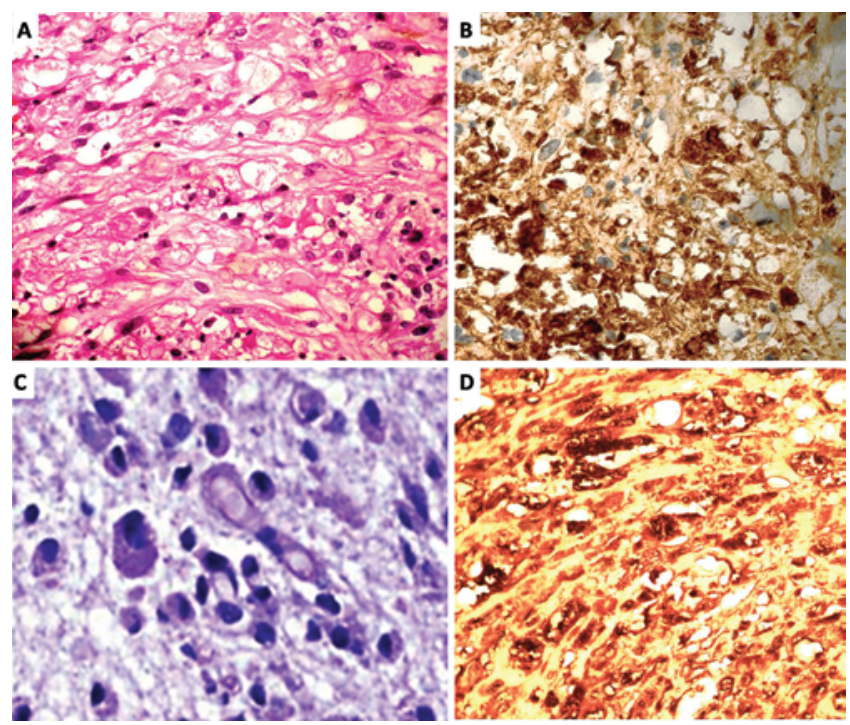

FIG. 2. A: Some of the tumor cells are markedly pleomorphic. They have enlarged hyperchromatic nuclei with frequent intranuclear pseudoinclusions, multinucleations, and inconspicuous nucleoli. Many eosinophilic granular bodies are visible, as are very sparse mitoses. There is no evidence of endothelial cell hyperplasia or necrosis. B: Cytoplasmic positivity for GFAP. C: Cytoplasmic positivity for PAS. D: Cytoplasmic positivity for $\mathrm{S} 100$. H \& E (A); original magnification $\times 200$ for all panels. Figure is available in color online only.

central nervous system. ${ }^{3}$ Most reported cases occur in the suprasellar region. The pineal localization is extremely rare, with only 4 previously reported cases in the literature. , $10,15^{1}$

The histogenesis of a GCT is debated. For tumors that arise from the peripheral nervous system, Schwann cells appear to be the most likely cells of origin. ${ }^{15}$ The source of a pineal region GCT may differ from its extracranial counterpart. For GCTs in the sellar area, the pituicyte, a modified astrocyte and the principal posterior pituitary cell, is considered the cell of origin., ${ }^{3,12,14}$ Some authors now consider the GCT as a hamartomatous lesion; and other authors, as tumors of uncertain histogenesis. ${ }^{13}$ To date, no recognized common cell accounts for all types of GCT.

The age of tumor occurrence in 4 cases, including our patient, was under 40 years, and only 1 patient was 67 years of age. The cases to date occurred in 3 males and 2 females. Symptoms were generally related to the compression of adjacent structures. The most frequent symptoms, including in our case, were dysfunctions of eye movements (Parinaud syndrome) and signs of rising intracranial tension caused by hydrocephalus (Table 1). Sudden-onset diplopia or visual loss, short-term history of confusion, headache, and vomiting have been described by Snipes et al. ${ }^{15}$ and Nitta et al. ${ }^{9}$ The duration of symptoms has ranged from sudden onset (5 days) to 4 years. Spontaneous intratumoral hemorrhage and intraventricular hemorrhage have also been reported in suprasellar region GCAs. ${ }^{3,14}$

The imaging findings of GCTs are relatively nonspecific given the broad range of attenuation and signal intensity of most common pineal region masses: germinoma, meningioma, pineocytoma, and glioma..$^{3,9,10,14,15}$ In our
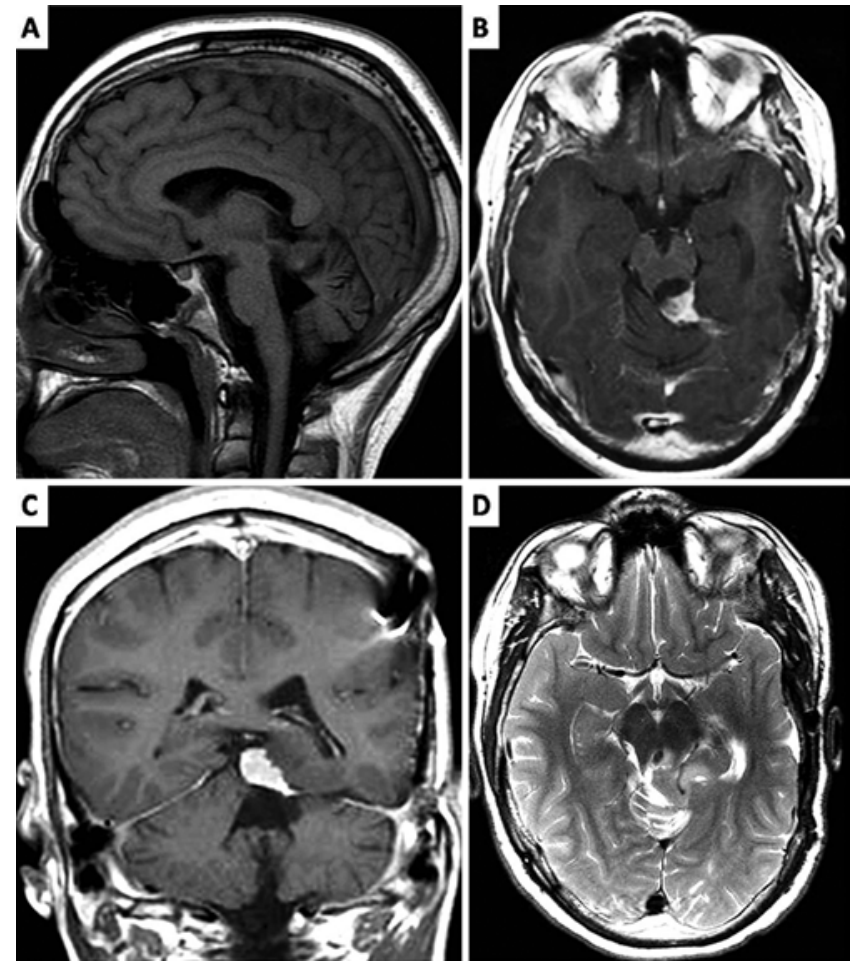

FIG. 3. Sagittal T1-weighted MR image without Gd (A), axial (B) and coronal (C) Gd-enhanced T1-weighted MR images, and axial T2weighted MR image (D) obtained 3 years postoperatively, showing a small residual tumor.

case and in the case reported by Ohta et al., the tumor presented as a well-demarcated, low-intensity to isointense lesion in the pineal body and third ventricle wall with homogeneous contrast enhancement. ${ }^{10}$ In these cases the CT and MRI characteristics were similar to the appearance of a large meningioma. Snipes et al. described the CT scanning features of a case with a cystic component. ${ }^{15}$ On the other hand, a GCA in the pineal region has often been associated with increased signal intensity in the adjacent thalami on MRI, while GCAs elsewhere promote virtually no edema in the adjacent brain structures. The presence of calcification, which was reported by Snipes et al., was exceptionally reported in other localizations. ${ }^{15}$

Granular cell tumor is generally a surprising finding in that it is seldom considered in the preoperative differential diagnosis of a pineal region lesion. ${ }^{3,9,10,15}$ They display a distinctive histological appearance characterized by the presence of neoplastic cells with large eosinophilic and granular cytoplasm. ${ }^{4,10,12,13}$ At the periphery of the tumor, gradual transition into classic astrocytoma is frequently found, although it can be subtle. ${ }^{6-9,12}$ Tumors can be entirely composed of granular cells, but more often granular cells coexist with conventional infiltrating astrocytoma. Immunohistochemical studies show that most GCAs are positive for GFAP and S100., Negative GFAP immunostaining was reported in a few articles.,,$_{3,8,10,12,13}$ As in our case, GCAs are positive for CD68, PAS, and EMA.

The treatment of pineal region tumors remains one of the major intellectual and technical challenges facing neurosurgeons. The choice of the surgical approach depends 
on the size and histological nature of the tumor and the neurosurgeon's habits. In our case, we used the subtemporal approach to achieve subtotal resection of the tumor. The parietooccipital interhemispheric transtentorial route was performed in the case described by Nitta et al., while the occipital transtentorial approach was used by Snipes et al. for subtotal resection in 2 cases and by Ohta et al. for total resection in 1 case. ${ }^{9,10,15}$ The firm and vascular nature of GCTs often encountered intraoperatively, along with the lack of an obvious dissection plane between the tumor and normal brain and adjacent vessels, often prohibits their gross-total resection. ${ }^{3,9,10,15}$ An attempt at subtotal resection of this slow-growing tumor may be justified in certain patients in whom gross-total removal was determined to be too risky at the time of surgery. In only 1 reported case, the tumor was considered soft and could be aspirated after radiation therapy.$^{15}$ Nitta et al. found no signs of recurrence for 7 years postoperatively without any adjuvant therapy. ${ }^{9}$ For this reason, we did not administer adjuvant therapy to our patient.

The natural history of a GCT is poorly understood. ${ }^{3,9,12}$ Although no systematic studies of this tumor have been undertaken to date, GCTs of the neurohypophysis and pineal region, unlike GCAs of the cerebral hemisphere, seem to be typically benign and indolent despite occasional invasion and recurrence. ${ }^{2,3,10}$ Follow-up evaluation in our case, in the 4 previously reported pineal cases, and in cases in all other intracranial localizations has shown that subtotal resection is effective for symptomatic tumors. ${ }^{6,8-10,12,15}$ The role of radiation therapy for this benign, slow-growing tumor remains unclear. Outcomes analysis of the published cases treated using subtotal resection and with available follow-up data showed no difference in recurrence between patients who received radiation treatment and those who did not. Glazer et al. described 1 patient who underwent 3 weeks of postoperative radiation therapy consisting of 32 Grays after subtotal resection. ${ }^{4}$ The patient died 3 months postoperatively. No radiation effect on the tumor was noted at autopsy. Therefore, in light of the tumor's slow growth, radiation therapy for these lesions remains controversial. ${ }^{3}$ However, adjuvant radiation treatment may be reasonable for tumors with atypical features. ${ }^{11}$

\section{Conclusions}

A GCT should be considered in the differential diagnosis of an enhancing lesion in the pineal region. This tumor is commonly firm and vascular. However, the surgeon must be prepared to face a hemorrhagic unresectable tumor. The goal of decompression of the surrounding structures via subtotal resection may be reasonable. Although this tumor has been known as an aggressive astrocytic entity, our review of the pineal localization revealed a relative benign tumor prognosis. Thus, in our opinion, GCA in the pineal region must be managed with less aggressive therapeutic management.

\section{Acknowledgment}

We thank Sophie Domingues-Montanari for her help with English editing.

\section{References}

1. Bonnin JM, Rubinstein LJ: Immunohistochemistry of central nervous system tumors. Its contributions to neurosurgical diagnosis. J Neurosurg 60:1121-1133, 1984

2. Brat DJ, Scheithauer BW, Medina-Flores R, Rosenblum MK, Burger PC: Infiltrative astrocytomas with granular cell features (granular cell astrocytomas): a study of histopathologic features, grading, and outcome. Am J Surg Pathol 26:750757,2002

3. Cohen-Gadol AA, Pichelmann MA, Link MJ, Scheithauer BW, Krecke KN, Young WF Jr, et al: Granular cell tumor of the sellar and suprasellar region: clinicopathologic study of 11 cases and literature review. Mayo Clin Proc 78:567-573, 2003

4. Glazer N, Hauser H, Slade H: Granular cell tumor of the neurohypophysis. Am J Roentgenol Radium Ther Nucl Med 76:324-326, 1956

5. Heyerdahl Strøm E, Skullerud K: Pleomorphic xanthoastrocytoma: report of 5 cases. Clin Neuropathol 2:188-191, 1983

6. Hirato J, Nakazato Y: Pathology of pineal region tumors. J Neurooncol 54:239-249, 2001

7. Kros JM, Vecht CJ, Stefanko SZ: The pleomorphic xanthoastrocytoma and its differential diagnosis: a study of five cases. Hum Pathol 22:1128-1135, 1991

8. Kumar P, Tatke M, Sharma A, Singh D: Histological analysis of lesions of the pineal region: a retrospective study of 12 years. Pathol Res Pract 202:85-92, 2006

9. Nitta J, Tada T, Kyoshima K, Goto T, Ishii K, Hongo K, et al: Atypical pleomorphic astrocytoma in the pineal gland: case report. Neurosurgery 49:1458-1461, 2001

10. Ohta T, Yachi K, Ogino A, Yokoyama T, Fukushima T, Watanabe T, et al: Pleomorphic granular cell astrocytoma in the pineal gland: case report. Neuropathology 30:615-620, 2010

11. Palma L, Celli P, Maleci A, Di Lorenzo N, Cantore G: Malignant monstrocellular brain tumours. A study of 42 surgically treated cases. Acta Neurochir (Wien) 97:17-25, 1989

12. Saad A, Mo J, Miles L, Witte D: Granular cell astrocytoma of the cerebellum: report of the first case. Am J Clin Pathol 126:602-607, 2006

13. Shi Y, Morgenstern N: Granular cell astrocytoma. Arch Pathol Lab Med 132:1946-1950, 2008

14. Shin E, Ki Chung C, Park SH: Granular cell astrocytoma. Pathol Res Pract 203:57-62, 2007

15. Snipes GJ, Horoupian DS, Shuer LM, Silverberg GD: Pleomorphic granular cell astrocytoma of the pineal gland. Cancer 70:2159-2165, 1992

\section{Author Contributions \\ Conception and design: El Asri, Baallal, Boucetta, El Mostarchid. Acquisition of data: El Asri, Sinaa, Gazzaz. Analysis and inter- pretation of data: El Asri. Drafting the article: Sinaa. Critically revising the article: all authors. Reviewed submitted version of manuscript: all authors. Approved the final version of the manu- script on behalf of all authors: El Asri.}

\section{Correspondence}

Abad Cherif El Asri, Department of Neurosurgery, Military Hospital, Rabat 10100, Morocco. email: abad20031@hotmail. com. 